Research Article

\title{
Some Variants of Krasnoselskii-Type Fixed Point Results for Equiexpansive Mappings with Applications
}

\author{
Niaz Ahmad, ${ }^{1}$ Nayyar Mehmood $\mathbb{D}^{1}{ }^{1}$ and Ahmed Al-Rawashdeh $\mathbb{D}^{2}$ \\ ${ }^{1}$ Department of Mathematics and Statistics, International Islamic University, H-10 Islamabad, Pakistan \\ ${ }^{2}$ Department of Mathematical Sciences, College of Science, UAE University, UAE \\ Correspondence should be addressed to Ahmed Al-Rawashdeh; aalrawashdeh@uaeu.ac.ae
}

Received 23 July 2021; Accepted 28 August 2021; Published 11 September 2021

Academic Editor: Pasquale Vetro

Copyright (C) 2021 Niaz Ahmad et al. This is an open access article distributed under the Creative Commons Attribution License, which permits unrestricted use, distribution, and reproduction in any medium, provided the original work is properly cited.

In this paper, we investigate the Krasnoselskii-type fixed point results for the operator $F$ of two variables by assuming that the family $\{F(x,): x$.$\} is equiexpansive. The results may be considered as variants of the Krasnoselskii fixed point theorem in a$ general setting. We use our main results to obtain the existence of solutions of a fractional evolution differential equation. An example of a controlled system is given to illustrate the application.

\section{Introduction}

Fractional evolution equations give a unique way to evaluate the well-posedness of many complicated systems. Differential models with fractional derivatives provide a great tool for the description of memory and hereditary properties. The fractional-order models of real physical systems are always more appropriate than the classical integer order systems. Many fractional order controlled problems and fractional evolution differential equations are recently studied. Their existence results can be seen in [1-5]. Most of the results involve contractive operators with more restrictive conditions. This is the reason that many existence results cover a restrictive class of physical problems.

Krasnoselskii fixed point theorem is a generalized form of Schauder and Banach fixed point theorems. While studying the solutions of delay and neutral differential/integral equations, it has been noticed that the solution can be expressed as a sum of contractive and compact operators. This theorem plays an important role in the existence of solutions of delay integral equations and neutral functional equations. Many generalizations and modifications of the Krasnoselskii fixed point theorem have appeared; for example, see [6-16] and the references therein. The Krasnoselskii fixed point theorem [17] may be stated as follows:
Theorem 1. Let $V$ be a Banach space and $\Omega$ be a closed convex nonempty subset of $V$. Suppose $\xi$ and $\zeta$ map $\Omega$ into $V$ such that

(1) $\xi v+\zeta v \in \Omega$ for all $v, v$ in $\Omega$

(2) $\xi$ is compact and continuous

(3) $\zeta$ is contraction mapping

Then, there is a $v$ in $\Omega$ such that $\xi v+\zeta v=v$.

In Krasnoselskii fixed point theorem, there are two operators in which $\xi$ is compact and continuous and $\zeta$ is a contraction mapping. It is important to note that there is a very restrict and small class of operators that are contractions; due to this reason, many theorems involving contractive operators are less applicable. Therefore, it is required to cover more applications with different types of operators; in this regard, one of the suitable choices is the class of expansive operators. Also, there are a number of equations in which we cannot decompose the operator as a sum of two or more operators. To overcome this situation, the authors in [9-11] established Krasnoselskii-type results in a more general setting consisting of a single map $\Gamma$ depending upon two variables. The following result may be seen in [11]: 
Theorem 2. Let $\Omega$ be a bounded closed convex subset of a given Banach space $X$, and $\Gamma$ be a mapping of $\Omega \times \Omega$ into $\Omega$ such that

(1) $\left\|\Gamma\left(\nu, v_{1}\right)-\Gamma\left(\nu, v_{2}\right)\right\| \leq \gamma\left\|v_{1}-v_{2}\right\|$ for some $\gamma \in[0,1)$, and for all $v \in X$

(2) $\left\|\Gamma\left(v_{1}, v\right)-\Gamma\left(v_{2}, v\right)\right\| \leq\left\|\xi v_{1}-\xi v_{2}\right\|$

where $\xi$ is completely continuous and maps $\Omega$ into $\Omega$. Then, there is an element $v \in \Omega$ such that $\Gamma(v, v)=v$.

If we define $\Gamma(v, v)=\xi v+\zeta v$ where $\xi$ is compact and continuous and $B$ is contraction then, Theorem 1 is the special case of Theorem 2 .

In most fixed point results, there is a condition such that $T(\Omega) \subseteq \Omega$, where $\Omega \subseteq X, X$ is a metric space and $T$ is an operator on $\Omega$ into $\Omega$. No much attention has been given to the case $\Omega \subseteq T(\Omega)$. Xiang and Yuan [15] investigated the case $\Omega \subseteq T(\Omega)$ and obtained the following important results in this direction.

Theorem 3 (see [15]). Let $\Omega$ be a nonempty closed subset of a complete metric space $X$ and $\zeta$ is an expansive mapping of $\Omega$ into $X$ such that $\Omega \subseteq \zeta(\Omega)$. Then, there is a unique $s \in \Omega$ such that $\zeta s=s$.

Theorem 4 (see [15]). Let $X$ be a Banach space and $\Omega$ be a nonempty closed convex subset of $X$. Suppose $\xi$ and $\zeta$ are mappings of $\Omega$ into $X$ such that

(1) $\xi$ is continuous such that $\xi(\Omega)$ lies in a compact subset of $X$

(2) $\zeta$ is an expansive mapping

(3) $\Omega \subseteq v+\zeta(\Omega)$ for $v \in \xi(\Omega)$

Then, there is $s \in \Omega$ with $\xi s+\zeta s=s$.

Definition 5 (see [9]). Let $X$ be a Banach space and $\Gamma: X \times$ $X \longrightarrow X$. The family $\{\Gamma(., v): v \in X\}$ is called equicontractive if there is a $k \in[0,1)$ such that

$$
\left\|\Gamma\left(v_{1}, v\right)-\Gamma\left(v_{2}, v\right)\right\| \leq k\left\|v_{1}-v_{2}\right\|,
$$

for all $\left(v_{1}, v\right),\left(v_{2}, v\right)$ in the domain of $\Gamma$.

In a similar way, we define the following.

Definition 6. Let $X$ be a Banach space and $\Gamma: X \times X \longrightarrow X$. The family $\{\Gamma(\nu,):. v \in X\}$ is called equiexpansive if there is $h>1$ such that

$$
\left\|\Gamma\left(\nu, v_{1}\right)-\Gamma\left(\nu, v_{2}\right)\right\| \geq h\left\|v_{1}-v_{2}\right\|,
$$

for all $\left(v, v_{1}\right),\left(v, v_{2}\right)$ in the domain of $\Gamma$.

\section{Main Results}

Inspired by the above results (Theorems 1, 2, 3, and 4), we study the Krasnoselskii-type fixed point results for the operator $\Gamma$ with conditions:

(i) The family $\{\Gamma(\nu,):. \nu \in X\}$ is equiexpansive

(ii) $\Omega \subseteq \Gamma(\nu, \Omega)$ for $v \in \Omega$ or $\Omega \subseteq \Gamma(v, \Omega)$ for $v \in \xi(\Omega)$

where $\Omega$ is a subset of a Banach space $X$ and $\xi$ is an operator of $\Omega$ into $\Omega$ or $X$.

Remark 7. In Theorem 2, the mapping $\Gamma$ is equicontractive and $\Gamma(v, v) \in \Omega$ for $v, v$ in $\Omega$ while in (i) and (ii), the family $\{\Gamma(\nu,): v$.$\} is equiexpansive and \Omega \subseteq \Gamma(v, \Omega)$ for $v \in \xi(\Omega)$.

Theorem 8. Suppose $\Omega$ be a nonempty closed convex subset of a Banach space $X$ and $\xi$ be a continuous mapping of $\Omega$ into $X$ such that $\xi(\Omega)$ resides in a compact subset of $X$. Let $\Gamma$ be a mapping of $\xi(\Omega) \times \Omega$ into $X$ such that the family $\{\Gamma(v,): v$. $\in \xi(\Omega)\}$ is equiexpansive and $\Omega \subseteq \Gamma(v, \Omega)$ for $v \in \xi(\Omega)$ with

$$
\left\|\Gamma\left(v_{1}, v\right)-\Gamma\left(v_{2}, v\right)\right\| \leq\left\|v_{1}-v_{2}\right\| .
$$

Then, there is $s \in \Omega$ such that $\Gamma(\xi s, s)=s$.

Proof. For $u \in \xi(\Omega)$, define a mapping $H$ of $\Omega$ into $X$ by $H$ $(v)=\Gamma(u, v)$. The mapping $H$ is expansive because

$$
\left\|H\left(v_{1}\right)-H\left(v_{2}\right)\right\|=\left\|\Gamma\left(u, v_{1}\right)-\Gamma\left(u, v_{2}\right)\right\| \geq h\left\|v_{1}-v_{2}\right\|,
$$

and $\Omega \subseteq \Gamma(v, \Omega)=H(\Omega)$. By Theorem 3, there exists a unique point in $s^{*} \in \Omega$ such that $H\left(s^{*}\right)=s^{*}$, since for each $u \in \xi(\Omega)$, there is a unique $s^{*} \in \Omega$, so we can define $F: \xi(\Omega)$

$\longrightarrow \Omega$ to obtain $\mathrm{F} v=s^{*}$ for some $v \in \xi(\Omega)$ ); therefore,

we have $\mathrm{F} v=\Gamma(v, \mathrm{~F} v)$. Now, for $v_{1}, v_{2} \in \xi(\Omega)$,

$$
\begin{aligned}
\left\|\mathrm{F}\left(v_{1}\right)=\mathrm{F}\left(v_{2}\right)\right\| & =\left\|\Gamma\left(v_{1}, \mathrm{~F} v_{1}\right)-\Gamma\left(v_{2}, \mathrm{~F} v_{2}\right)\right\| \\
& =\left\|\left(\Gamma\left(v_{2}, \mathrm{~F} v_{1}\right)-\Gamma\left(v_{2}, \mathrm{~F} v_{2}\right)\right)-\left(\Gamma\left(v_{2}, \mathrm{~F} v_{1}\right)-\Gamma\left(v_{1}, \mathrm{~F} v_{1}\right)\right)\right\| \\
& \geq\left\|\Gamma\left(v_{2}, \mathrm{~F} v_{1}\right)-\Gamma\left(v_{2}, \mathrm{~F} v_{2}\right)\right\|-\left\|\Gamma\left(v_{2}, \mathrm{~F} v_{1}\right)-\Gamma\left(v_{1}, \mathrm{~F} v_{1}\right)\right\| \\
& \geq h\left\|\mathrm{~F} v_{1}-\mathrm{F} v_{2}\right\|-\left\|v_{1}-v_{2}\right\| .
\end{aligned}
$$

This means that

$$
\left\|\mathrm{F} v_{1}-\mathrm{F} v_{2}\right\| \leq \frac{1}{h-1}\left\|v_{1}-v_{2}\right\|, \quad h>1,
$$

which shows that $F$ is a continuous function of $\xi(\Omega)$ into $\Omega$. Also, $\mathrm{F} \circ \xi$ is a continuous function of $\Omega$ into $\Omega$. Since $\xi(\Omega) \subseteq C$, where $C$ is any compact set; therefore, $\xi(\bar{\Omega}) \subseteq \bar{C}$ $=C$ and $\xi(\Omega)$ is compact by [18] (page 37). By [19] (page 412), $\mathrm{F}(\xi(\Omega))$ is compact because $\xi \Omega$ is relatively compact, and $\mathrm{F}(\xi(\Omega))$ resides in a compact set $\mathrm{F}(\xi(\Omega))$. Since $\mathrm{F}(\xi(\Omega)) \subseteq \Omega$, therefore, $\mathrm{F}(\xi \overline{(}(\Omega)) \subseteq \bar{\Omega}=\Omega$. Hence, $\mathrm{F} \circ \xi(\Omega)$ $=\mathrm{F}(\xi(\Omega)) \subseteq \mathrm{F}(\bar{\xi}(\Omega))=\mathrm{F} \circ \bar{\xi}(\Omega) \subseteq \Omega$ and $\mathrm{F} \circ \xi$ is a continuous function of $\Omega$ into a compact set $\mathrm{F} \circ \bar{\xi}(\Omega) \subseteq \Omega$. By 
Schauder second theorem [17], there is $s \in \Omega$ such that $\mathrm{F}^{\circ}$ $\xi(s)=s$ or $\mathrm{F}(\xi s)=s$. Since for $\xi s \in \xi(\Omega)$ there is unique $\mathrm{F}(\xi s) \in \Omega$ such that $\Gamma(\xi s, \mathrm{~F}(\xi s))=\mathrm{F}(\xi s)$ and also $\mathrm{F}(\xi s)=s$, therefore, $\Gamma(\xi s, s)=s$.

Corollary 9 (see [15]). Let $X$ be a Banach space and $\Omega$ be a nonempty closed convex subset of $X$. Suppose $\xi$ and $\zeta$ are mappings of $\Omega$ into $X$ such that

(1) $\xi$ is continuous such that $\xi(\Omega)$ lies in a compact subset of $X$

(2) $\zeta$ is an expansive mapping

(3) $\Omega \subseteq v+\zeta(\Omega)$ for $v \in \xi(\Omega)$

Then, there is $s \in \Omega$ with $\xi s+\zeta s=s$.

Proof. Define $\Gamma(v, v)=v+\zeta v$ with $v=\xi v \in \xi(\Omega)$ (Theorem 8). Since $\Gamma(v, \Omega)=v+\zeta(\Omega)$ and from (3) $\Omega \subseteq v+\zeta(\Omega)$, therefore, $\Omega \subseteq \Gamma(v, \Omega)$. Now, since $\zeta$ is expansive mapping, therefore,

$$
\left\|\Gamma\left(v, v_{1}\right)-\Gamma\left(v, v_{2}\right)\right\|=\left\|\zeta v_{1}-\zeta v_{2}\right\| \geq h\left\|v_{1}-v_{2}\right\|
$$

Also,

$$
\left\|\Gamma\left(v_{1}, v\right)-\Gamma\left(v_{2}, v\right)\right\|=\left\|v_{1}-v_{2}\right\| \leq\left\|v_{1}-v_{2}\right\| .
$$

Hence, there is $s \in \Omega$ such that $s=\Gamma(\xi s, s)=\xi s+\zeta s$.

Remark 10. The above corollary is Theorem 2.2 in [15] or above Theorem 4 . If we take $\xi=0$, zero operator, then, we obtain Theorem 3 or Theorem 2.1 in [15].

Corollary 11. Let $X$ be a Banach space and $\Omega$ be a nonempty closed convex subset of $X$. Suppose $\xi$ and $\zeta$ are mappings of $\Omega$ into $X$ such that

(1) $\xi$ nonexpansive and $\xi(\Omega) \subseteq \Omega$ lies in a compact subset of $X$

(2) $\zeta$ is expansive mapping

(3) $\Omega \subseteq \xi v+\zeta(\Omega)$ for $v \in \xi(\Omega)$

Then, there is $s \in \Omega$ with $(\xi \circ \xi) s+\zeta s=s$.

Proof. Define $\Gamma(v, v)=\xi v+\zeta v$ with $v=\xi v \in \xi(\Omega)$ (Theorem 8). Since $\Gamma(v, \Omega)=\xi v+\zeta(\Omega)$ and from $(3) \Omega \subseteq \xi v+\zeta(\Omega)$ for $v \in \xi(\Omega)$, therefore, $\Omega \subseteq \Gamma(v, \Omega)$. Now, since $\zeta$ is expansive mapping, therefore,

$$
\left\|\Gamma\left(v, v_{1}\right)-\Gamma\left(v, v_{2}\right)\right\|=\left\|\zeta v_{1}-\zeta v_{2}\right\| \geq h\left\|v_{1}-v_{2}\right\| .
$$

Also, from (1), $\xi$ is continuous and

$$
\left\|\Gamma\left(v_{1}, v\right)-\Gamma\left(v_{2}, v\right)\right\|=\left\|\xi v_{1}-\xi v_{2}\right\| \leq\left\|v_{1}-v_{2}\right\| .
$$

Hence, there is $s \in \Omega$ such that $s=\Gamma(\xi s, s)=(\xi \circ \xi) s+\zeta s$.
Theorem 12. Suppose $\Omega$ be a nonempty closed convex set such that $\Omega$ lies in a compact subset of a Banach space $X . \Gamma$ be a mapping of $\Omega \times \Omega$ into $X$ such that the family $\{\Gamma(\nu,)$. $: v \in \Omega\}$ is equiexpansive and $\Omega \subseteq \Gamma(\nu, \Omega)$ for $v \in \Omega$. Let $\xi$ be a continuous mapping of $\Omega$ into a metric space $Y$ with

$$
\left\|\Gamma\left(v_{1}, v\right)-\Gamma\left(v_{2}, v\right)\right\| \leq d_{v}\left(\xi v_{1}, \xi v_{2}\right)
$$

Then, there is $s \in \Omega$ such that $\Gamma(s, s)=s$.

Proof. For $v \in \Omega$, define a mapping $H$ of $\Omega$ into $X$ by $H(v)$ $=\Gamma(v, v)$. The mapping $H$ is expansive and $\Omega \subseteq \Gamma(v, \Omega)=$ $H(\Omega)$. By (Theorem 3 ), there exists a unique point in $\Omega$ say $\mathrm{F} v$ such that $\mathrm{F} v=\Gamma(\nu, F v)$. Now,

$$
\begin{aligned}
\left\|\mathrm{F}\left(v_{1}\right)-\mathrm{F}\left(v_{2}\right)\right\| & =\left\|\Gamma\left(v_{1}, \mathrm{~F} v_{1}\right)-\Gamma\left(v_{2}, \mathrm{~F} v_{2}\right)\right\| \\
& =\left\|\left(\Gamma\left(v_{2}, \mathrm{~F} v_{1}\right)-\Gamma\left(v_{2}, \mathrm{~F} v_{2}\right)\right)-\left(\Gamma\left(v_{2}, \mathrm{~F} v_{1}\right)-\Gamma\left(v_{1}, \mathrm{~F} v_{1}\right)\right)\right\| \\
& \geq\left\|\Gamma\left(v_{2}, \mathrm{~F} v_{1}\right)-\Gamma\left(v_{2} \mathrm{~F}\right)\right\|-\left\|\Gamma\left(v_{2}, \mathrm{~F} v_{1}\right)-\Gamma\left(v_{1}, \mathrm{~F} v_{1}\right)\right\| \\
& \geq h\left\|\mathrm{~F} v_{1}-\mathrm{F} v_{2}\right\|-d_{v}\left(\xi v_{1}, \xi v_{2}\right) .
\end{aligned}
$$

This means that

$$
\left\|\mathrm{F} \nu_{1}-\mathrm{F} v_{2}\right\| \leq \frac{1}{h-1} d_{v}\left(\xi \nu_{1}, \xi \nu_{2}\right), \quad h>1
$$

In the above expression, the continuity of $\xi$ implies the continuity of $\mathrm{F}$. Since $\Omega$ lies in a compact subset of $X$ and $F$ is continuous, therefore, $F(\Omega)$ lies in a compact subset of $\Omega$. So by Schauder second theorem [17], there is $s \in \Omega$ such that $\mathrm{F} s=s$. Since for $s \in \Omega$ there is unique $\mathrm{F} s \in \Omega$ such that $\Gamma(s, \mathrm{~F} s)=\mathrm{F} s$ and also $\mathrm{F} s=s$, therefore, $\Gamma(s, s)=s$.

Corollary 13. Let $\Omega$ be a nonempty closed convex set such that $\Omega$ lies in a compact subset of a Banach space $X . \Gamma$ be a mapping of $\Omega \times \Omega$ into $X$ such that

(1) $\Omega \subseteq \Gamma(\nu, \Omega)$ for $v \in \Omega$

(2) $\left\|\Gamma\left(\nu, v_{1}\right)-\Gamma\left(\nu, v_{2}\right)\right\| \geq h\left\|v_{1}-v_{2}\right\|, h>1$

(3) $\left\|\Gamma\left(v_{1}, v\right)-\Gamma\left(v_{2}, v\right)\right\| \leq\left\|\xi v_{1}-\xi v_{2}\right\|$

where $\xi$ be a continuous mapping of $\Omega$ into $X$; then, there is $s \in \Omega$ such that $\Gamma(s, s)=s$.

Proof. Take $v=X$ (where $X$ is a Banach space) in the above theorem.

Remark 14. The above corollary is a variant of Theorem 2 or Theorem 8 in [11].

Corollary 15. Let $X$ be a Banach space and $\Omega$ be a nonempty closed convex set such that $\Omega$ lies in a compact subset of $X$. Suppose $\xi$ and $\zeta$ are mappings of $\Omega$ into $X$ such that

(1) $\xi$ is a continuous mapping

(2) $\zeta$ is an expansive mapping 
(3) $\Omega \subseteq \xi v+\zeta(\Omega)$ for $v \in \Omega$

Then, there is $s \in \Omega$ such that $\xi s+\zeta s=s$.

Proof. Define $\Gamma(v, v)=\xi v+\zeta v$ (in the above Corollary 13). Since $\Gamma(\nu, \Omega)=\xi v+\zeta(\Omega)$ and from (3) $\Omega \subseteq \xi v+\zeta(\Omega)$, therefore, $\Omega \subseteq \Gamma(\nu, \Omega)$. Now, since $\zeta$ is expansive mapping, therefore,

$$
\left\|\Gamma\left(\nu, v_{1}\right)-\Gamma\left(\nu, v_{2}\right)\right\|=\left\|\zeta v_{1}-\zeta v_{2}\right\| \geq h\left\|v_{1}-v_{2}\right\| .
$$

Also,

$\left\|\Gamma\left(\nu_{1}, v\right)-\Gamma\left(\nu_{2}, v\right)\right\|=\left\|\xi \nu_{1}-\xi v_{2}\right\| \leq\left\|\xi v_{1}-\xi \nu_{2}\right\|$

Hence, there is $s \in \Omega$ such that $s=\Gamma(s, s)=\xi s+\zeta s$.

Theorem 16. Suppose $\Omega$ be a nonempty closed convex subset of a Banach space $X$ and $\xi$ be a continuous mapping of $\Omega$ into $\Omega$ such that $\xi(\Omega)$ is contained in a compact subset of $X$. Let $\Gamma$ be a mapping of $\xi(\Omega) \times \Omega$ into $X$ such that the family $\{\Gamma(v,):. v \in \xi(\Omega)\}$ equiexpansive and $\Omega \subseteq \Gamma(v, \Omega)$ for $v \in \xi(\Omega)$ with

$$
\left\|\Gamma\left(v_{1}, v\right)-\Gamma\left(v_{2}, v\right)\right\| \leq\left\|\xi v_{1}-\xi v_{2}\right\|
$$

Then, there exists $s \in \Omega$ such that $\Gamma(\xi s, s)=s$.

Proof. For $u \in \xi(\Omega)$, define a mapping $H$ of $\Omega$ into $X$ by $H$ $(v)=\Gamma(u, v)$. The mapping $H$ is expansive because

$$
\left\|H\left(v_{1}\right)-H\left(v_{2}\right)\right\|=\left\|\Gamma\left(v, v_{1}\right)-\Gamma\left(v, v_{2}\right)\right\| \geq h\left\|v_{1}-v_{2}\right\| .
$$

Also, $\Omega \subseteq \Gamma(v, \Omega)=H(\Omega)$. By Theorem 3, there exists a unique point say $\mathrm{F} v$ such that $\mathrm{F} v=\Gamma(v, \mathrm{~F} v)$. Now,

$$
\begin{aligned}
\left\|\mathrm{F}\left(v_{1}\right)-\mathrm{F}\left(v_{2}\right)\right\| & =\left\|\Gamma\left(v_{1}, \mathrm{~F} v_{1}\right)-\Gamma\left(v_{2}, \mathrm{~F} v_{2}\right)\right\| \\
& =\left\|\left(\Gamma\left(v_{2}, \mathrm{~F} v_{1}\right)-\Gamma\left(v_{2}, \mathrm{~F} v_{2}\right)\right)-\left(\Gamma\left(v_{2}, \mathrm{~F} v_{1}\right)-\Gamma\left(v_{1}, \mathrm{~F} v_{1}\right)\right)\right\| \\
& \geq\left\|\Gamma\left(v_{2}, \mathrm{~F} v_{1}\right)-\Gamma\left(v_{2}, \mathrm{~F} v_{2}\right)\right\|-\left\|\Gamma\left(v_{2}, \mathrm{~F} v_{1}\right)-\Gamma\left(v_{1}, \mathrm{~F} v_{1}\right)\right\| \\
& \geq h\left\|\mathrm{~F}\left(v_{1}\right)-\mathrm{F}\left(v_{2}\right)\right\|-\left\|\xi v_{1}-\xi v_{2}\right\| .
\end{aligned}
$$

This means that

$$
\left\|\mathrm{F} v_{1}-\mathrm{F} v_{2}\right\| \leq \frac{1}{h-1}\left\|\xi v_{1}-\xi v_{2}\right\|, \quad h>1
$$

Since $\xi$ is continuous, so $F$ is a continuous function of $\xi(\Omega)$ into $\Omega$. Also, $\mathrm{F} \circ \xi$ is a continuous function of $\Omega$ into $\Omega$ and $F \circ \xi(\Omega)$ resides in a compact subset of $\Omega$. So by Schauder second theorem [17], there is $s \in \Omega$ such that $\mathrm{F} \circ \xi(s)=s$ or $\mathrm{F}(\xi s)=s$. Since for $\xi s \in \xi(\Omega)$ there is unique $\mathrm{F}(\xi s) \in \Omega$ such that $\Gamma(\xi s, \mathrm{~F}(\xi s))=\mathrm{F}(\xi s)$ and also $\mathrm{F}(\xi s)=s$, therefore, $\Gamma(\xi s, s)=s$.

Remark 17. In the above theorem, $\xi$ is a mapping of $\Omega$ into $\Omega$ while in Theorem $8, \xi$ is a mapping of $\Omega$ into $X$.
Corollary 18. Let $X$ be a Banach space and $\Omega$ be a nonempty closed convex subset of X. Suppose $\xi$ is a mapping of $\Omega$ into $\Omega$ and $\zeta$ is mapping of $\Omega$ into $X$ such that

(1) $\xi$ is continuous such that $\xi(\Omega)$ lies in a compact subset of $X$

(2) $\zeta$ is an expansive mapping

(3) $v \in \xi(\Omega)$ implies $\Omega \subseteq \xi v+\zeta(\Omega)$

Then, there exists $s \in \Omega$ with $(\xi \circ \xi) s+\zeta s=s$.

Proof. Taking $\Gamma(u, v)=\xi u+\zeta v$ with $v=\xi u \in \xi(\Omega)$ (in the above Theorem 16), we can obtain the required result.

Theorem 19. Suppose $\Omega$ be a nonempty closed convex subset of a Banach space $X$ and $\xi$ be a continuous mapping of $\Omega$ into $Y$ such that $\xi(\Omega)$ is a precompact subset of a metric space $Y$. Let $\Gamma$ be a continuous mapping of $\xi(\bar{\Omega}) \times \Omega$ into $X$ such that the family $\{\Gamma(v,):. v \in \xi(\Omega)\}$ equiexpansive and $\Omega \subseteq \Gamma(v, \Omega)$ for $v \in \xi(\bar{\Omega})$. Then, there is $s \in \Omega$ such that $\Gamma(\xi s, s)=s$.

Proof. For $u \in \xi(\bar{\Omega})$, define a mapping $H$ of $\Omega$ into $X$ by $H$ $(v)=\Gamma(u, v)$. The mapping $H$ is expansive because

$$
\left\|H\left(v_{1}\right)-H\left(v_{2}\right)\right\|=\left\|\Gamma\left(u, v_{1}\right)-\Gamma\left(u, v_{2}\right)\right\| \geq h\left\|v_{1}-v_{2}\right\| .
$$

Also, $\Omega \subseteq \Gamma(u, \Omega)=H(\Omega)$. By Theorem 3, there is a unique point in $\Omega$ say $\mathrm{F} v$ such that $\mathrm{F} v=\Gamma(v, \mathrm{~F} v)$. We show that the mapping $\mathrm{F}$ of $\xi(\bar{\Omega})$ into $\Omega$ is continuous. Let $v_{n}$ be a sequence in $\xi(\bar{\Omega})$ such that $v_{n}$ converges to $v_{0}$ in $\xi(\bar{\Omega})$. Then,

$$
\begin{aligned}
\left\|\mathrm{F}\left(v_{n}\right)-\mathrm{F}\left(v_{0}\right)\right\|= & \left\|\Gamma\left(v_{n}, \mathrm{~F}\left(v_{n}\right)\right)-\Gamma\left(v_{0}, \mathrm{~F}\left(v_{0}\right)\right)\right\| \\
= & \|\left(\Gamma\left(v_{n}, \mathrm{~F}\left(v_{n}\right)\right)-\Gamma\left(v_{n}, \mathrm{~F}\left(v_{0}\right)\right)\right) \\
& -\left(\Gamma\left(v_{0}, \mathrm{~F}\left(v_{0}\right)\right)-\Gamma\left(v_{n}, \mathrm{~F}\left(v_{0}\right)\right)\right) \| \\
\geq & \left\|\Gamma\left(v_{n}, \mathrm{~F}\left(v_{n}\right)\right)-\Gamma\left(v_{n}, \mathrm{~F}\left(v_{0}\right)\right)\right\| \\
& -\left\|\Gamma\left(v_{0}, \mathrm{~F}\left(v_{0}\right)\right)-\Gamma\left(v_{n}, \mathrm{~F}\left(v_{0}\right)\right)\right\| \\
\geq & \left.h \| \mathrm{F}\left(v_{n}\right)-\mathrm{F}\left(v_{0}\right)\right) \| \\
& -\left\|\Gamma\left(v_{0}, \mathrm{~F}\left(v_{0}\right)\right)-\Gamma\left(v_{n}, \mathrm{~F}\left(v_{0}\right)\right)\right\|,
\end{aligned}
$$

and therefore,

$$
\left\|\mathrm{F}\left(v_{n}\right)-\mathrm{F}\left(v_{0}\right)\right\| \leq \frac{1}{h-1}\left\|\Gamma\left(v_{0}, \mathrm{~F}\left(v_{0}\right)\right)-\Gamma\left(v_{n}, \mathrm{~F}\left(v_{0}\right)\right)\right\| .
$$

Since $\Gamma$ is continuous, therefore, $F$ is continuous. The operator $F$ maps $\xi(\bar{\Omega})$ into $\Omega$ and is continuous. Since $\xi(\Omega)$ is relatively compact, therefore, by [19] (page 412), $\mathrm{F}(\xi(\Omega))$ is relatively compact. Let $\operatorname{co}(\mathrm{F}(\xi(\Omega)))$ be the convex hull of $\mathrm{F}(\xi(\Omega))$. By [20] (page 195), $\operatorname{co}(\mathrm{F}(\xi(\Omega))$ ) is relatively compact. Since $\Omega$ is closed and convex, so $\operatorname{co}(\mathrm{F}(\bar{\xi}(\Omega))) \subseteq$ $\Omega$, and by [21] (page 415), $\overline{c o}(\mathrm{~F}(\xi(\Omega))) \subseteq \Omega$. Let $P=\overline{c o}(\mathrm{~F}$ $(\xi(\Omega)))$; then, $P$ is a subset of $\Omega$ and $F \circ \xi(P) \subseteq P$. Since 
$P=\operatorname{co}(\mathrm{F}(\bar{\xi}(\Omega)))$ also, therefore, $P$ is compact and convex. So by first Schauder theorem [17], there is a point $s \in P$ such that $\mathrm{F}(\xi s)=s$. Thus, $s=\Gamma(\xi s, s)$ by similar arguments given in the proof of Theorem 5 .

Remark 20. The above theorem is a variant of Theorem 2.2 in [9].

\section{Application}

Let $X$ be a Banach space with norm $\|\cdot\|$, for $\xi>0$ denote $J=[0, \xi]$ a closed interval in $\mathbb{R}$. Let $C(J, X)$ be the Banach space of all continuous functions from $J$ into $X$ with norm $\|v\|=\sup _{\rho \in J}|v(\rho)|$, where $v \in C(J, X)$. Let $U$ be a closed and convex subset of $X$. Consider the following controlled system:

$$
\left\{\begin{array}{l}
D^{\alpha} v(\mathrm{\varrho})=-\xi v(\mathrm{\varrho})+\Gamma(\mathrm{\varrho}, v(\mathrm{\varrho}))+C(\mathrm{\varrho}) v(\mathrm{\varrho}), \mathrm{\varrho} \in J:=[0, \xi], v \in U, \text { and } \alpha \in(0,1), \\
v(0)=v_{0} .
\end{array}\right.
$$
[22]:

The mild solution corresponding to $v$ of $(\xi)$ is given by

$$
\begin{aligned}
v^{v}(\varrho)= & \aleph(\varrho) v_{0}+\int_{0}^{\varrho}(\rho-s)^{\alpha-1} \mathfrak{\Im}(\varrho-s) \Gamma(s, v(s)) d s \\
& +\int_{0}^{\varrho}(\rho-s)^{\alpha-1} \mathfrak{\Im}(\rho-s) C(s) v(s) d s,
\end{aligned}
$$

where

$$
\begin{aligned}
& \aleph(\varrho)=\int_{0}^{\infty} \xi_{\alpha}(\theta) T\left(\mathrm{\varrho}^{\alpha} \theta\right) d \theta, \\
& \Im(\varrho)=\alpha \int_{0}^{\infty} \theta \xi_{\alpha}(\theta) T\left(\mathrm{\varrho}^{\alpha} \theta\right) d \theta,
\end{aligned}
$$

$\xi_{\alpha}$ is the probability density function defined on $(0, \infty)$; for more details about the notations, we refer the readers to [22]. Also,

$$
\begin{aligned}
\|T(\mathrm{\varrho})\| & \leq \Omega \text { for some } \Omega>1, \\
\|\aleph(\mathrm{\varrho}) v\| & \leq \Omega\|v\|, \\
\|\Im(\mathrm{\varrho}) v\| & \leq \frac{\alpha \Omega}{\Gamma(\alpha+1)}\|v\|,
\end{aligned}
$$

and $\aleph(\varrho)$ and $\mathfrak{I}(\varrho)$ are strongly continuous for $\mathrm{Q} \geq 0$ and are compact for each $\mathrm{Q}>0$.

We can write $(\zeta)$ in the form

$$
\Gamma(v, v)(\mathrm{\varrho})=\aleph(\mathrm{\varrho}) v_{0}+\int_{0}^{\mathrm{\varrho}}(\mathrm{\varrho}-s)^{\alpha-1} \mathfrak{\Im}(\mathrm{\varrho}-s) \Gamma(s, v(s)) d s+Q(v),
$$

where

$$
Q(v)(\mathrm{e})=\int_{0}^{\varrho}(\mathrm{Q}-s)^{\alpha-1} \mathfrak{\Im}(\mathrm{\varrho}-s) C(s) v(s) d s .
$$

Assume that the following conditions hold:

$(H 1) Q: \zeta_{\delta} \longrightarrow X$ is continuous and expansive, i.e., $\| Q($ $v)-Q(v)\|\geq \ell\| v-v \|$ for all $v, v \in \zeta$ and some $\ell>1$

$(H 2) \Gamma: Q\left(\bar{\zeta}_{\delta}\right) \times \zeta_{\delta} \longrightarrow X$ is continuous

$(H 3) \zeta_{\delta} \subseteq \Gamma\left(v, \zeta_{\delta}\right)$ for each $v \in Q\left(\bar{\zeta}_{\delta}\right)$

where $\zeta_{\delta}$ is a closed ball with radius $\delta$ and center at 0 in $X$.

We show that $\Gamma$ is equiexpansive. For a fixed $v$, and any $v, u \in Q\left(\bar{\zeta}_{\delta}\right)$, consider

$$
\begin{aligned}
& \|\Gamma(v, v)-\Gamma(v, u)\| \\
& =\left\|\begin{array}{l}
\aleph(\mathrm{\varrho}) v_{0}+\int_{0}^{\varrho}(\mathrm{\varrho}-s)^{\alpha-1} \mathfrak{\Im}(\mathrm{\varrho}-s) \Gamma(s, v(s)) d s+Q(v) \\
-\aleph(\mathrm{\varrho}) v_{0}-\int_{0}^{\varrho}(\varrho-s)^{\alpha-1} \mathfrak{\Im}(\mathrm{\varrho}-s) \Gamma(s, v(s)) d s-Q(u)
\end{array}\right\| \\
& =\|Q(v)-Q(u)\| \geq \ell\|v-u\| \text {, }
\end{aligned}
$$

which shows that $\left\{\Gamma(v, \cdot): v \in Q\left(\bar{\zeta}_{\delta}\right)\right\}$ is equiexpansive.

We prove compactness of $Q$. Continuity of $\mathfrak{I}$ and $C$ implies the continuity of $Q$; we show that $Q$ is uniformly bounded on $\zeta_{\delta}$. For $v \in \zeta_{\delta}$, consider

$$
\begin{aligned}
\|Q(v)(\varrho)\| & =\left\|\int_{0}^{\varrho}(\varrho-s)^{\alpha-1} \mathfrak{\Im}(\mathrm{\varrho}-s) C(s) v(s) d s\right\| \\
& \leq \int_{0}^{\varrho}(\varrho-s)^{\alpha-1}\left\|\xi^{q} \mathfrak{\Im}(\mathrm{\varrho}-s)\right\|\|C(s)\|\|v(s)\| d s \\
& \leq \frac{\|C\| \Omega_{q} \alpha \Gamma(2-q)}{\Gamma(1+\alpha(1-q))} \delta \int_{0}^{\varrho}(\mathrm{\varrho}-s)^{\alpha-1} d s \\
& \leq \frac{\|C\| \Omega_{q} \alpha \Gamma(2-q)}{\Gamma(1+\alpha(1-q))} \delta \frac{T^{\alpha(1-q)}}{\alpha(1-q)}:=\mathrm{\varrho},
\end{aligned}
$$

which implies that $Q$ is uniformly bounded. To prove the equicontinuity of $Q$, we consider for $0<\mathrm{Q}_{1}<\mathrm{Q}_{2}<T$,

$$
\begin{aligned}
\left\|Q(v)\left(\mathrm{\varrho}_{2}\right)-Q(v)\left(\mathrm{\varrho}_{1}\right)\right\|= & \| \int_{0}^{\mathrm{\varrho}_{2}}\left(\mathrm{\varrho}_{2}-s\right)^{\alpha-1} \mathfrak{\Im}\left(\mathrm{\varrho}_{2}-s\right) C(s) v(s) d s \\
& -\int_{0}^{\varrho_{1}}\left(\mathrm{\varrho}_{1}-s\right)^{\alpha-1} \mathfrak{\Im}\left(\mathrm{\varrho}_{1}-s\right) C(s) v(s) d s \| \\
\leq & \frac{\|C\| \Omega_{q} \alpha \Gamma(2-q)}{\Gamma(1+\alpha(1-q))} \delta \mid \int_{0}^{\varrho_{2}}\left(\mathrm{\varrho}_{2}-s\right)^{\alpha-1} d s \\
& -\int_{0}^{\varrho_{1}}\left(\varrho_{1}-s\right)^{\alpha-1} d s \mid \\
\leq & \frac{\|C\| \Omega_{q} \Gamma(2-q)}{\Gamma(1+\alpha(1-q))} \delta\left|\varrho_{2}^{\alpha}-\varrho_{1}^{\alpha}\right| \longrightarrow 0 \text { as } \varrho_{2} \longrightarrow \mathrm{\varrho}_{1},
\end{aligned}
$$


which is independent of $v$, and $\left\|Q(v)\left(\varrho_{2}\right)-Q(v)\left(\varrho_{1}\right)\right\| \longrightarrow 0$ as $\mathrm{Q}_{2} \longrightarrow \mathrm{Q}_{1}$. Therefore, $Q$ is equicontinuous. By ArzelaAscoli theorem, $Q$ is a compact operator. From (H3), $\zeta_{\delta} \subseteq \Gamma\left(v, \zeta_{\delta}\right)$ for each $v \in Q\left(\bar{\zeta}_{\delta}\right)$. All conditions of Theorem 19 are satisfied to obtain the $z \in X$ such that $\Gamma(Q z, z)=z$, which is the solution of controlled problem $(\xi)$.

Finally, we summarize the above discussion in the following theorem.

Theorem 21. Let $X$ be a Banach space and $\zeta_{\delta}$ be a closed ball with center at 0 and radius $\delta$. Assume that $Q: \zeta_{\delta} \longrightarrow X$ and $\Gamma: Q\left(\bar{\zeta}_{\delta}\right) \times \zeta_{\delta} \longrightarrow X$ satisfy $(H 1)-(H 3)$. Then, there exists a mild solution of controlled problem $(\xi)$.

Example 22. Consider the following controlled system:

$$
\left\{\begin{array}{l}
\frac{\partial^{\alpha} v(\mathrm{\varrho}, v)}{\partial \mathrm{\varrho}^{\alpha}}=\frac{\partial^{2} v(\mathrm{\varrho}, v)}{\partial v^{2}}+\Gamma(\mathrm{\varrho}, v(\mathrm{\varrho}, v))+\int_{0}^{\varrho} \mathrm{F}(s, v(s, v)) d s \\
v(\mathrm{\varrho}, 0)=v(\mathrm{\varrho}, \pi)=0, v^{\prime}(\mathrm{\varrho}, 0)=v^{\prime}(\mathrm{\varrho}, \pi)=0 \\
v(0, v)+\Sigma \xi_{i} v\left(\varrho_{i}, v\right)=v_{0}(v)
\end{array}\right.
$$

where $\varrho \in J=[0,1], v \in(0, \pi), 0<\alpha<1$, let $X=L^{2}([0, \pi])$ and consider the operator $\xi: D(\xi) \subseteq X \longrightarrow X$ defined by

$$
\xi(v)=\frac{\partial^{2} v}{\partial v^{2}}
$$

with domain

$$
D(\xi)=\left\{v, \frac{\partial v}{\partial v}, \frac{\partial^{2} v}{\partial v^{2}} \in X\right\}
$$

Clearly, $\xi$ is densely defined in $X$ and is the infinitesimal generator of a resolvent family $\left\{T_{\alpha}(\varrho)\right\}_{\rho \geq 0}$ on $X[4]$ and let $v, v \in C(J, X)$. Define the operators $\Gamma, \mathrm{F}: J \times U \longrightarrow X$ by

$$
\begin{gathered}
\Gamma(\varrho, v)=\frac{\left(1+e^{\varrho}\right)(1+|v(\varrho, v)|)}{e^{-\varrho}|v(\varrho, v)|}, \\
\mathrm{F}(\varrho, v)=2 v(\varrho, v) .
\end{gathered}
$$

Clearly,

$$
|\Gamma(\mathrm{\varrho}, v)-\Gamma(\mathrm{\varrho}, v)| \geq\left(1+e^{\varrho}\right)|v-v|,
$$

which shows that $\Gamma$ is equiexpansive. Note that $\xi$ and $F$ are continuous and compact. All conditions of Theorem 21 are satisfied to obtain the solution of the given controlled problem.

\section{Conclusion}

Some variants of the Krasnoselskii-type fixed point theorem are presented using the notion of newly defined equiexpan- sive mappings. As an application, we present an existence result for controlled problem with less conditions. This problem is also a generalized kind of Cauchy problem for evolution differential equations.

\section{Data Availability}

No data were used to support the results; the results are proved completely using mathematical proofs.

\section{Conflicts of Interest}

The authors declare that they have no conflicts of interest.

\section{Acknowledgments}

The third author gratefully acknowledges with thanks the Department of Research Affairs at UAEU. This article is supported by the grant: UPAR-2019, Fund No. 31 S397.

\section{References}

[1] M. Bouaouid, K. Hilal, and S. Melliani, "Sequential evolution conformable differential equations of second order with nonlocal condition," Advances in Difference Equations, vol. 2019, no. 1, 2019.

[2] M. Mohan Raja and V. Vijayakumar, "New results concerning to approximate controllability of fractional integro-differential evolution equations of order $1<\mathrm{r}<2$.," Numerical Methods for Partial Differential Equations., vol. 18, 2020.

[3] M. M. Raja, V. Vijayakumar, and R. Udhayakumar, "Results on the existence and controllability of fractional integrodifferential system of order $1<\mathrm{r}<2$ via measure of noncompactness," Chaos, Solitons \& Fractals, vol. 139, p. 110299, 2020.

[4] X. B. Shu and Q. Wang, "The existence and uniqueness of mild solutions for fractional differential equations with nonlocal conditions of order $1<\alpha<2$," Computers \& Mathematics with Applications., vol. 64, no. 6, pp. 2100-2110, 2012.

[5] W. K. Williams, V. Vijayakumar, R. Udhayakumar, and K. S. Nisar, "A new study on existence and uniqueness of nonlocal fractional delay differential systems of order $1<\mathrm{r}<2$ in Banach spaces," Numerical Methods for Partial Differential Equations., vol. 7, 2020

[6] A. Azizi, R. Moradi, and A. Razani, "Expansive mappings and their applications in modular space," In Abstract and applied analysis, vol. 2014, article 580508, pp. 1-8, 2014.

[7] T. A. Burton, "A fixed-point theorem of Krasnoselskii," Applied Mathematics Letters., vol. 11, no. 1, pp. 85-88, 1998.

[8] Y. Z. Chen, "Krasnoselskii-type fixed point theorems using $\alpha$ -concave operators," Journal of Fixed Point Theory Applications., vol. 22, p. 52, 2020.

[9] G. L. Karakostas, “An extension of Krasnoselski's fixed point theorem for contractions and compact mappings," Topological Methods in Nonlinear Analysis., vol. 22, no. 1, pp. 181-191, 2003.

[10] W. R. Melvin, "Some extensions of the Krasnoselskii fixed point theorems," Journal of Differential Equations, vol. 11, no. 2, pp. 335-348, 1972.

[11] M. Z. Nashed and J. S. Wong, "Some variants of a fixed point theorem of Krasnoselskii and applications to nonlinear 
integral equations," Journal of Mathematics and Mechanics., vol. 18, no. 8, pp. 767-777, 1969.

[12] E. Pourhadi, R. Saadati, and Z. Kadelburg, "Some Krasnosel'skii-type fixed point theorems for Meir-Keelertype mappings," Nonlinear Analysis: Modelling and Control., vol. 25, no. 2, pp. 257-265, 2020.

[13] S. Park, "Generalizations of the Krasnoselskii fixed point theorem," Nonlinear Analysis: Theory, Methods \& Applications., vol. 67, no. 12, pp. 3401-3410, 2007.

[14] D. Wardowski, "Family of mappings with an equicontractivetype condition," Journal of Fixed Point Theory and Applications., vol. 22, p. 55, 2020.

[15] T. Xiang and R. Yuan, “A class of expansive-type Krasnosel'skii fixed point theorems," Nonlinear Analysis: Theory, Methods \& Applications., vol. 71, no. 7-8, pp. 3229-3239, 2009.

[16] T. Xiang and R. Yuan, "Critical type of Krasnosel'skii fixed point theorem," Proceedings of the American Mathematical Society., vol. 139, no. 3, pp. 1033-1044, 2011.

[17] D. R. Smart, Fixed Point Theorems, Cup Archive, 1980.

[18] W. Rudin, Principles of Mathematical Analysis, McGraw-hill, New York, 1964.

[19] E. Kreyszig, Introductory functional analysis with applications, wiley, New York, 1978.

[20] J. H. Shapiro, A Fixed-Point Farrago, Springer, 2016.

[21] N. Dunford and J. T. Schwartz, Linear Operators, Part 1: General Theory, John Wiley \& Sons, 1988.

[22] J. Wang and Y. Zhou, "A class of fractional evolution equations and optimal controls," Nonlinear Analysis: Real World Applications., vol. 12, no. 1, pp. 262-272, 2011. 